\title{
NUMERICAL SIMULATION OF CRATER CREATING PROCESS IN DYNAMIC REPLACEMENT METHOD BY SMOOTH PARTICLE HYDRODYNAMICS
}

\author{
ANDRZEJ DANILEWICZ, ZBIGNIEW SIKORA \\ Gdańsk University of Technology, Department of Geotechnics, Geology and Maritime Engineering, Gdańsk, Poland, \\ e-mail: adanilew@pg.gda.pl,zbig@pg.gda.pl
}

\begin{abstract}
A theoretical base of SPH method, including the governing equations, discussion of importance of the smoothing function length, contact formulation, boundary treatment and finally utilization in hydrocode simulations are presented. An application of SPH to a real case of large penetrations (crater creating) into the soil caused by falling mass in Dynamic Replacement Method is discussed. An influence of particles spacing on method accuracy is presented. An example calculated by LS-DYNA software is discussed. Chronological development of Smooth Particle Hydrodynamics is presented. Theoretical basics of SPH method stability and consistency in SPH formulation, artificial viscosity and boundary treatment are discussed. Time integration techniques with stability conditions, SPH+FEM coupling, constitutive equation and equation of state (EOS) are presented as well.
\end{abstract}

Key words: dynamic replacement method, SPH, impact soil

\section{INTRODUCTION}

The objective of this paper is to propose a numerical methodology for simulating Dynamic Replacement Method process, actually its first stage - crater creating (Fig. 1).

The proposed methodology - hybrid Smooth Particle Hydrodynamics SPH+FEM is a combination of two numerical methods. First, the Smooth Particle Method is a discretization methodology that works without any grid or mesh, therefore it belongs to the mesh-free family methods. SPH was originally developed by Lucy [2], Gingold and Monaghan [3] initially for astrophysical problems. The reason for developing SPH method (and other meshless methods) is its capability for describing problems involving large deformation, fragmentation, cracks. However, the problems involving large deformation are important in many field of geotechnics. Smooth Particle Hydrodynamics - mesh free Lagrangian method was adapted to solid mechanics by Libersky and Petschek [5] and improved over the years.

Some works related to large deformation problems in soil using SPH method were made by Bojanowski and Kulak [6], [7], Fasanella et al. [9], Danilewicz [8], Kulak and Schwer [10].

In this paper, some basics of Smooth Particle Hydrodynamics with governing equations (importance of smoothing function and smoothing length, stability and consistence in SPH formulation, artificial viscosity and boundary treatment), descriptions due to constitutive equation, equations of state (EOS), together with an example of crater creating calculated in LS-DYNA will be presented.

\section{DYNAMIC REPLACEMENT METHOD}

Dynamic Replacement method (DRM) is a common method for soil improvement, when significant thickness of the improved soil $(5-7 \mathrm{~m})$ is to be realized. DRM uses impact energy to the ground by means of dropping a large mass from a significant height, that is applied in soft, saturated cohesive soils and soft organic soils. Dynamic Replacement offers effective means for increasing stiffness of the soil by increasing the average modulus of the inclusions within a soil mass resulting in much greater global modulus in the ground in comparison with the soil before stabilization. The technique involves the creation of craters in the ground platform from impacts by the falling mass (picture 2 in Fig. 1) and subsequently backfilling these holes with non-cohesive material such as crushed rock or concrete, gravel. The process is 

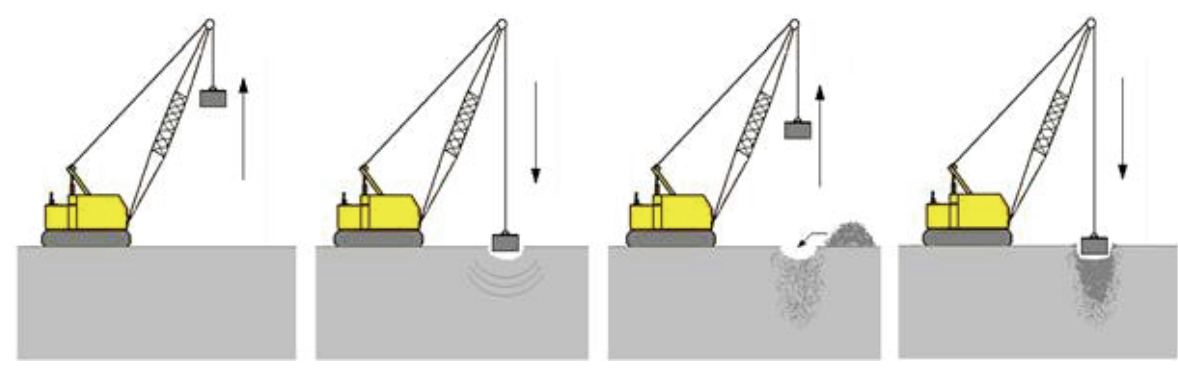

Fig. 1. Dynamic Replacement Method process (source: Keller Poland web page)

repeated to create large diameter columns, 1.5-4 meters within the soil mass, which besides introducing high modulus inclusions into the ground also creates drainage paths within the soils. Columns can be formed to the depth of $7 \mathrm{~m}$, beyond which the lateral spread at the bases of the columns counteracts further penetration. Dynamic Replacement offers a very cost competitive method of ground improvement. Benefits are also simplicity and short time of realization. The process of improving soil properties (modulus) requires knowledge of geotechnical ground profiles, proper matching of pounder mass, its shape and size, falling mass height, number of impacts on every grid node, and much more related information such as energy on surface unit or velocity of a pounder at impact time. All this information allows us to design the proper soil improvement by the DR method.

The aim of this paper is to propose a methodology to simulate a crater creating process. Knowledge of the influence of this process is important in proper DRM design especially in column grid design.

\section{BASICS OF SPH METHOD}

Hereafter an application to large deformation analysis of subsoil is presented (Danilewicz [8]).

The foundation of Smooth Particle Hydrodynamics is an interpolation theory. SPH interpolation is defined on a set of points without any grid or mesh. SPH utilises the following concept of interpolation at given particle-point " $i$ ", (Hiermaier [1])

$$
<u\left(x_{i}, t\right)>=\int_{R^{d}} u\left(x_{j}, t\right) W_{h}\left(\left|x_{i}-x_{j}\right|\right) d x_{j},
$$

where $W_{h}$ is so called kernel function or smoothing function and it is at least $C^{2}$ class. The particle approximation uses particles within the influence domain (of radius $\kappa h$, where $h$ is a smoothing length) of the smoothing function for a given particle $i$ (Fig. 2). Each $W_{h}$ function has to satisfy the following properties (Hiermaier [1]):
- normalization condition

$$
\int_{\Omega \subset R^{3}} W\left(x_{i}-x_{j}, h\right) d \Omega_{x_{j}}=1,
$$

- delta function property

$$
\lim _{h \rightarrow \infty} W\left(x_{i}-x_{j}, h\right)=\delta\left(x_{i}-x_{j}\right),
$$

- compact support

$$
W\left(x_{i}-x_{j}\right)=0 \text { for }\left|x_{i}-x_{j}\right|>\kappa h, \kappa \geq 1 .
$$

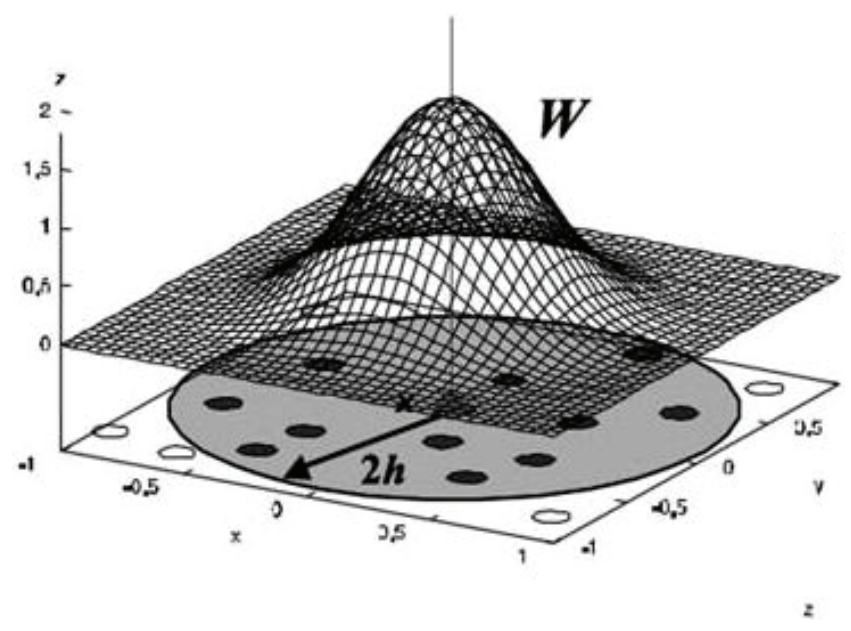

Fig. 2. Kernel function $W$. Reprint from Hiermaier [1]

Some possible kernel functions as, e.g., Gauss function

$$
W_{h}(u)=\frac{1}{\left(\pi h^{2}\right)^{n / 2}} \exp \left(\frac{-u^{2}}{h^{2}}\right)
$$

can also represent a possible kernel function.

Cubic B-spline proposed by Monaghan and Lattanzio [4]

$$
W\left(\frac{r}{h}\right)=\frac{C}{h^{d}}\left\{\begin{array}{cc}
1-3 / 2 q^{2}+3 / 4 q^{3} & 0 \leq q \leq 1, \\
1 / 4(2-q)^{3} & 1 \leq q \leq 2, \\
0 & q>2,
\end{array}\right.
$$


where $q=r / h, C=2 / 3,10 / 7 \pi$ or $1 / \pi$ for, 1,2 or 3 dimensions, respectively, can also be effectively applied as the appropriate kernel function. Scaling $r=\left|x_{i}-x_{j}\right|$ guarantees one particle SPH per smoothing length $h$.

Dividing the continuum into NP volumes and associating with appropriate masses, each volume element with its mass can be assigned to a particular SPH element, therefore discrete kernel approximation is to be written as (Hiermaier [1])

$$
<u_{i}>=\sum_{j=1}^{N P} u_{j} W_{h}\left(\left|x_{i}-x_{j}\right|\right) \frac{m_{j}}{\rho_{j}} .
$$

This form of approximation is directly used as a strong form for approximation of the PDE, therefore the approximation of derivatives is crucial in SPH method [4]

$$
<\nabla \cdot u(x, t)>=-\int_{R^{d}} u\left(x^{\prime}, t\right) \cdot \nabla W_{h}\left(\left|x-x^{\prime}\right|\right) d x^{\prime},
$$

where $d=1,2$ or 3 in the case of 1,2 or 3 dimensions.

\subsection{CONSERVATION EQUATIONS}

Using a given SPH approximation for the derivates one obtains the discrete conservation equation forms, for a given $i$ particle, as follows (after Li and Liu [11]):

- conservation of mass

$$
\frac{d \rho_{i}}{d t}=\rho_{i} \sum_{j=1}^{N P} \frac{m_{j}}{\rho_{j}}\left(v_{i}-v_{j}\right) \cdot \frac{\partial W_{i j}}{\partial x_{i}},
$$

- momentum equation

$$
\frac{d v_{i}}{d t}=-\sum_{j=1}^{N P} m_{j}\left(\frac{\sigma_{i}}{\rho_{i}^{2}}+\frac{\sigma_{j}}{\rho_{j}^{2}}\right) \frac{\partial W_{i j}}{\partial x_{i}},
$$

- energy equation

$$
\frac{d E_{i}}{d t}=\frac{1}{2} \sum_{j=1}^{N P} m_{j}\left(\frac{\sigma_{i}}{\rho_{i}^{2}}+\frac{\sigma_{j}}{\rho_{j}^{2}}\right): v_{i j} \frac{\partial W_{i j}}{\partial x_{i}} .
$$

\subsection{ARTIFICIAL VISCOSITY}

In dynamic impact simulation, loads are transmitted through the structure by shock waves. To prevent non-physical oscillations in velocity field and pressure beyond the shock wave in SPH method, some adjustments have to be done. The origins of such numerical instabilities are either due to discontinuity in velocity fields or algorithmic pathology in approximation of parabolic or hyperbolic systems ( $\mathrm{Li}$ and Liu [11]). By adding artificial viscosity equations to conservation equation it is possible to smear out shock wave over a few particles (distance bigger than the smoothing length). First proposed by Neumann and Richtmayer [12] and adopted to SPH method by Monaghan and Gingold [3], artificial viscosity takes on the following form

$$
\Pi_{i j}=\left\{\begin{array}{cc}
\frac{-\alpha_{\Pi} \bar{c}_{i j} \mu_{i j}+\beta_{\Pi} \mu_{i j}^{2}}{\bar{\rho}_{i j}} & v_{i j} \cdot x_{i j}<0, \\
0 & v_{i j} \cdot x_{i j} \geq 0,
\end{array}\right.
$$

where

$$
\begin{gathered}
\mu_{i j}=\frac{h v_{i j} \cdot x_{i j}}{x_{i j}^{2}+\varepsilon_{0} h_{i j}^{2}} ; \\
h_{i j}=\frac{1}{2}\left(h_{i}+h_{j}\right) ; \\
\bar{c}_{i j}=\frac{1}{2}\left(c_{i}+c_{j}\right) ; \\
\bar{\rho}_{i j}=\frac{1}{2}\left(\rho_{i}+\rho_{j}\right) ; \\
v_{i j}=v_{i}-v_{j} ; \quad x_{i j}=x_{i}-x_{j} ;
\end{gathered}
$$

with $\varepsilon_{0}$ usually equal 0.01 (Monagham [13]), $\alpha_{\Pi}, \beta_{\Pi}$ being viscosity parameters.

Therefore, e.g., the corrected momentum equation becomes

$$
\frac{d v_{i}}{d t}=-\sum_{j=1}^{N P} m_{j}\left(\frac{\sigma_{i}}{\rho_{i}^{2}}+\frac{\sigma_{j}}{\rho_{j}^{2}}+\Pi_{i j}\right) \frac{\partial W_{i j}}{\partial x_{i}} .
$$

\subsection{BOUNDARY TREATMENT}

Integration of a function in SPH approximation for the given $i$ particle has to be done inside the compact support. This requirement leads to some difficulties which can occur when inside the radius $\kappa h$ no sufficient number of neighbor particles exist. This can occur on the boundary. The solution is a so-called ghost particle approach (Fig. 3) proposed by Libersky and Petschek [5]. For a given $i$ SPH particle, with a distance from BC less than $\kappa h$, virtual SPH particles are symmetrically placed on the opposite side of BC. The same densities and velocities with opposite sign are assigned to virtual SPH. The Leonard-Jones molecular force produces repulse force to particles with posi- 
tion close to the boundary to prevent these particles from penetration.
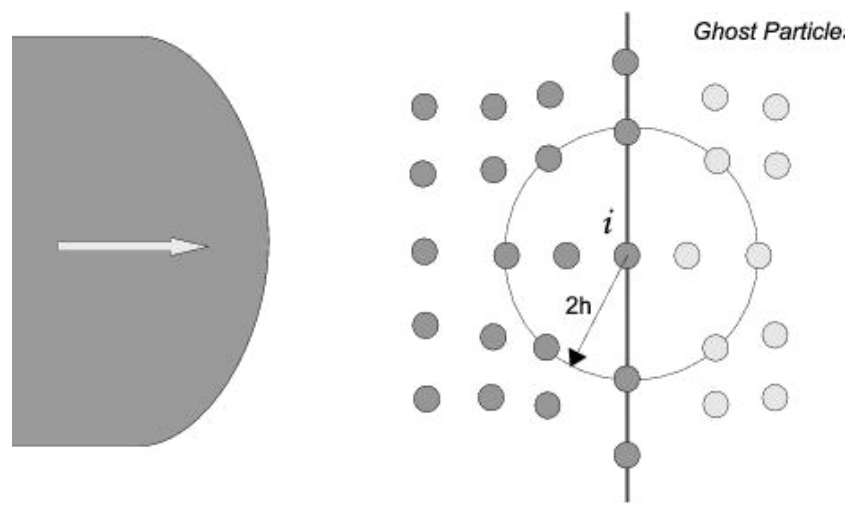

Fig. 3. Ghost particle approach in SPH method

\subsection{TIME INTEGRATION}

SPH method like other hydrocodes needs explicit method for time integration. The most widely used is Störmer-Verlet algorithm well known as leap-frog (LF) method. Stability of LF method is controlled by Courant-Friedrichs-Lewy condition, one of the proposed requirements for a maximum time step is $(\mathrm{Li}$ and Liu [11])

$$
\Delta t \leq C_{C F L} \frac{h}{c+s},
$$

where $c$ is the sound velocity, $h$ is a smoothing length and $s$ is a maximum particle speed over the whole particle set.

\subsection{SPH+FEM COUPLING}

Four main coupling types of mesh-free SPH and finite element method can be applied (Hiermaier [1]):

- master-slave coupling (contact algorithm),

- transition elements with boundary nodes that are at the same time SPH elements,

- hybrid coupling with an extension of the kernel approximation by components from nodes inside the kernel support,

- adaptive coupling that can start with certain discretization but can also convert some parts of discretization into more adequate one upon certain criteria.

Commercial hydrocodes software LS-DYNA was used for calculations. The loop of SPH cycle is presented in Fig. 4.

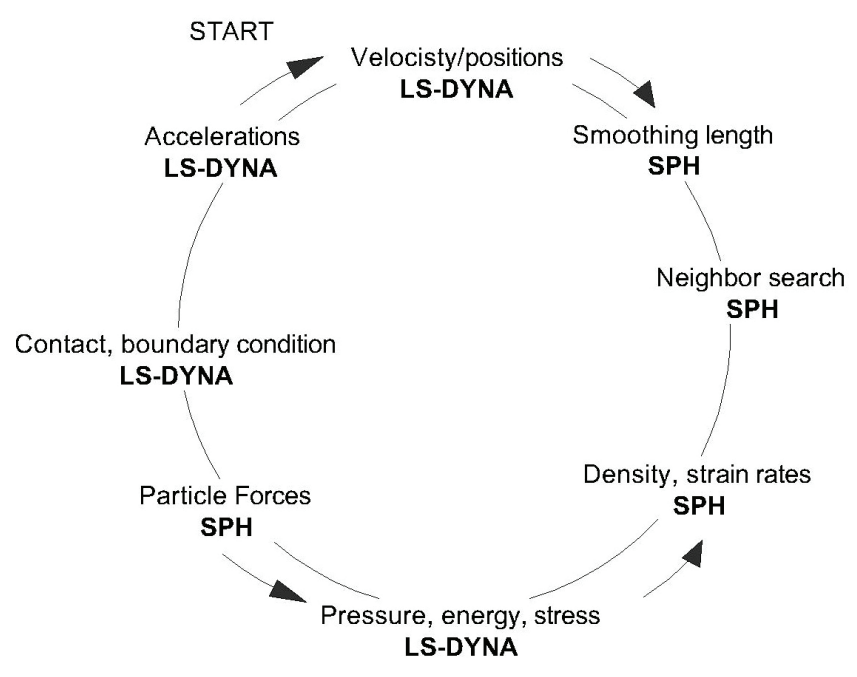

Fig. 4. The calculation cycle for SPH formulation in LS-DYNA software

\section{CONSTITUTIVE EQUATION}

All material models available in LS-DYNA software were tested in literature in the cases of large displacement and impact on soil (Thomas et al. [14], An [15], Heymsfield and Fasanella 2008, Kulak and Schwer [10]). The MAT005 chosen by the authors is a relatively simple elastic-plastic model with pressure dependent parabolic yield function with linear isotropic hardening.

$$
F(\sigma)=(p-f)\left(\Pi_{s}-\left(a_{0}+a_{1} p+a_{2} p\right)\right),
$$

where $p$ is a pressure, $a$ and $h$ are constants, $f$ is a function of the mean total strain and $\Pi_{s}$ is the second invariant of the stress deviator. Deviatoric stress rate has the form

$$
\dot{s}_{i j}=2 G \dot{e_{i j}}-\left(\frac{2 G s_{i j}: e_{i j}-\left(a_{1}+2 a_{2}\right) \dot{p}}{s_{i j}: s_{i j}}\right) s_{i j},
$$

where $e_{i j}$ is the deviatoric strain rate.

Yield surface is a surface of revolution centered on the hydrostat and increasing in radius with increasing pressure. The additional planar end cap is added (normal to the hydrostatic axis and also movable due to volumetric hardening).

As mentioned in Section 3.2, information about the load applied to the structure is transmitted through the continuum by waves and accordingly at the speed of sound in media discussed. Equation of state is a relation between pressure and volume at a given temperature. It is necessary to determine the pressure 
in shock compressed solid. The most popular ones (for structures under crash and impact) are MieGrüneisen equation of state and polynomial equation of state (after An [15]).

The relation between pressure and volumetric strain is determined by a tabulated curve and it is included in material input data. Thanks to this curve initial pressure required in SPH formulation can be easily obtained and therefore additional equation of state is not necessary.

\section{NUMERICAL EXAMPLE}

The geometry of numerical model is presented in Fig. 5. The falling rigid mass (modeled: $1.8 \mathrm{~m}$ in height and 0.46-0.6 m, width) was made from steel. The velocity at the soil surface was $7 \mathrm{~m} / \mathrm{s}$. Soil model has a quarter cylindrical shape of radius $4.8 \mathrm{~m}$, height of $6.8 \mathrm{~m}$ and it is surrounded by elastic enhancement made to represent semi-infinite soil domain. To save computational effort a hybrid SPH+FEM model was used and portion of soil expecting large deformation was modeled using 47.242 SPH elements, and the rest of the model was modeled using 5232 finite elements.

Soil constitutive parameters for material formulation MAT 005 (soil) are listed in Table 1. Material data was adopted from paper of Bojanowski and Kulak [6].

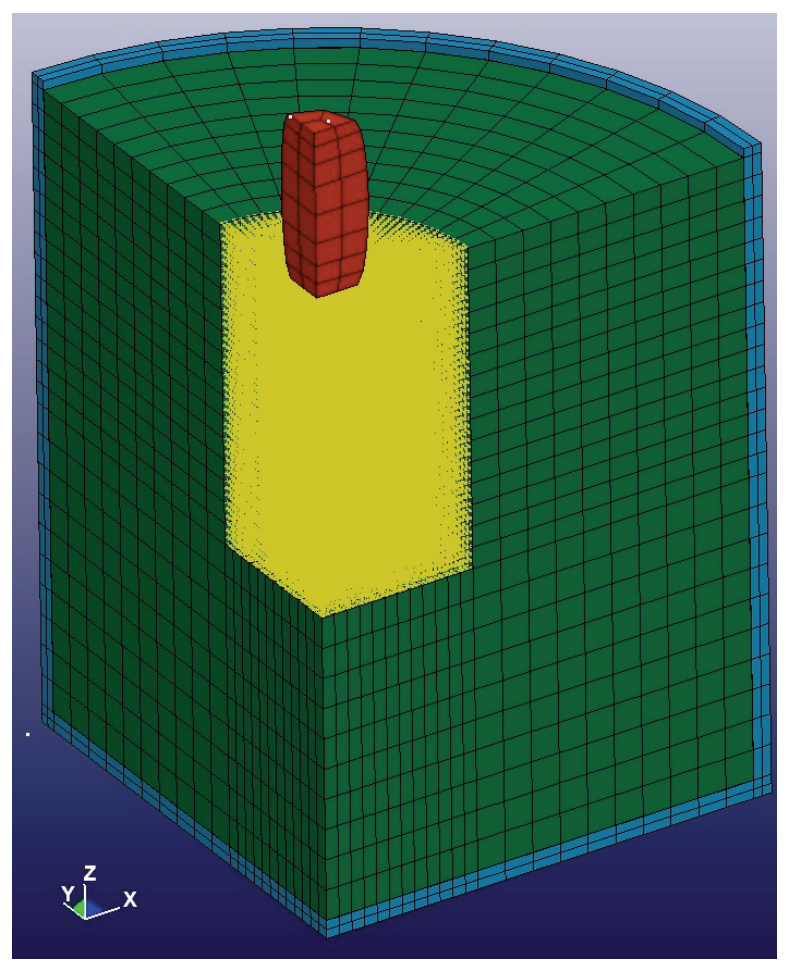

Fig. 5. Numerical hybrid SPH+FEM model for crater creating process by Dynamic Replacement Method
Table 1

Mat 005 material calibration (mm-s-tonne)

\begin{tabular}{|l|c|}
\hline \multicolumn{1}{|c|}{ Parameter } & Value \\
\hline Mass density & $2.35 \mathrm{E}-9$ \\
\hline Shear modulus & 34.474 \\
\hline Bulk modulus for unloading & 15.024 \\
\hline Yield function constant a0 & 0 \\
\hline Yield function constant a1 & 0 \\
\hline Yield function constant a2 & 0.602 \\
\hline Pressure cutoff for tensile fracture & 0 \\
\hline Volumetric crushing option & off \\
\hline
\end{tabular}

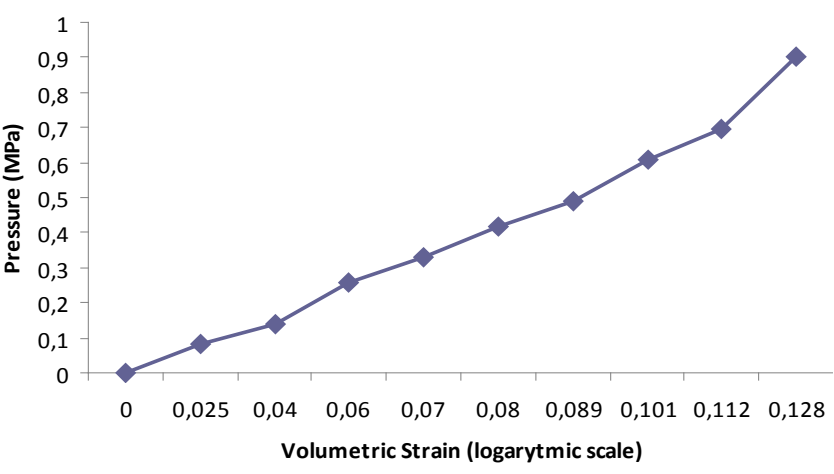

Fig. 6. Volumetric strain vs. pressure curve from hydrostatic compression

\section{RESULTS AND DISCUSSION}

The contour of effective stress is presented in Fig. 7. Hybrid SPH+FEM method needs much more computational effort than, e.g., FEM in Lagrange formulation, but mesh distortion (and problems with hour-

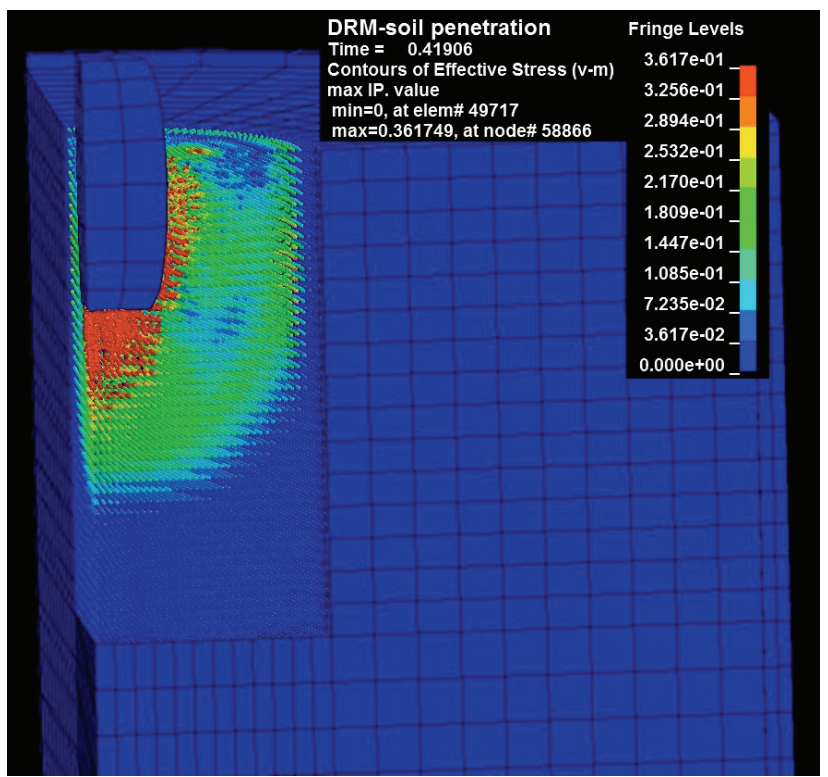

Fig. 7. Contours of effective stress at time $0.419 \mathrm{~s}$ after impact 
glassing) in this second one disqualifies it from practical viewpoint. This kind of numerical problem in the case of large deformation does not exist in SPH formulation. For a large penetration problem caused by rigid mass falling in Dynamic Replacement Method, more applicable is the SPH+FEM formulation (Danilewicz [8]). The authors were not able to perform validation with the model test at the time of writing this paper.

The validation procedure is to be performed in real condition.

\section{ACKNOWLEDGMENTS}

The authors wish to acknowledge the Polish National Science Center (grant No. 2011/01/N/ST8/07495) for its support. The discussion and correspondence with Dr. Bojanowski (Argonne National Laboratory, USA) was valuable. Calculations were carried out at the Academic Computer Center in Gdańsk.

\section{REFERENCES}

[1] Hiermaier S., Structures Under Crash and Impact, Springer, 2008.

[2] LuCY L.B., A Numerical Approach to The Testing of The Fission Hypothesis, Astronomical Journal, 1977, 82, 1013-1024.

[3] Monaghan J.J., Gingold R.A., Smoothed Particle Hydrodynamics: Theory and Applications to Non-Spherical Stars, Royal Astronomical Society, 1977, 181, 275-389.

[4] Monaghan J.J., Lattanzio J.C., A Refined Particle Mathod for Astrophysical Problems, Astronomy and Astrophysics, 1985, 149, 135-143.
[5] Libersky L.D., PetscheK A.G., Smooth Particle Hydrodynamics With Strength of Materials, Proceedings of the Next Free-Lagrange Conference, Moran, WY, USA, 1990, 347, 248-267.

[6] Bojanowski C., KULAK R., Comparsion of Lagrangian, SPH and MM-ALE approaches for Modeling Large Deformation in Soil, 11th International LS-DYNA Users Conference, 2010.

[7] Bojanowski C., Kulak R., Modeling of Cone Penetration Test Using SPH and MM-ALE Approaches, 8th European LS-DYNA Users Conference, 2011.

[8] Danilewicz A., Smooth Particle Hydrodynamics (SPH) Approach in Simulating Large Penetration Into Soil, Proceedings of European Young Geotechnical Engineers Conference, Gothenburg 2012.

[9] Fasanella E.L., Lyle R.K.H., Jackson K.E., Developing Soil Models for Dynamic Impact Simulations, NASA Langley Research Center, Hampton USA.

[10] Kulak R.F., Schwer L., Effect of Soil Material Models on SPH Simulations for Soil-Structure Interaction, Proceedings of 12 th International LS-DYNA Users Conference, 2012.

[11] Li S., LiU W.K., Meshfree Particle Methods, Springer, 2004.

[12] Von Neumann J., Richtmyer R.D., A Method for Numerical Calculation of Hydrodynamic Shocks, Journal of Applied Physics, 1950, 21, 232-237.

[13] Monaghan J.J., Simulating Free Surface Flows With SPH, Journal of Computational Physics, 1994, 399-406.

[14] Thoms M.A., ChitTy E.C., GildeA M.L., T'Kindt C.M., Constitutive Soil Properties for Cuddeback Lake, California and Carson Sink, Nevada, Raport NASA/CR-2008-215345, NASA Langley Research Center's (LaRC), 2008.

[15] JiCHONG AN, Soil Behavior under Blast Loading, PhD thesis, University of Nebraska, Lincoln 2010. 